\title{
Hard and fast, but within limits: is there a trade-off of stroke volume index and diastolic pressure in paediatric resuscitation?
}

RachaelK.Gregson1凹,2 Phone020 7905 2382Emailr.gregson@ucl.ac.uk

SophieSkellett2

SamiranRay 1,2

MarkJ.Peters1,2

1Respiratory, Critical Care and Anaesthesia (RCCA) Section in Infection, Immunity and Inflammation (III) Programme, UCL Great Ormond Street Institute of Child Health London UK

2 Paediatric and Neonatal Intensive Care, Great Ormond Street Hospital NHS

Foundation Trust London UK

Dear Editor,

The aim of chest compressions in cardiopulmonary resuscitation (CPR) is to restore organ perfusion while maintaining adequate coronary perfusion. The effects of force and rate of compressions are derived from animal studies; human data, especially from children, are scarce. A 3.5-year-old, 22-kg boy with ARDS received chest compressions for hypoxic cardiac arrest. Diastolic blood pressure (DBP, $\mathrm{mmHg}$ ) was measured invasively and stroke volume index $\left(\mathrm{SVI}, \mathrm{mL} / \mathrm{m}^{2}\right)$ was estimated using LiDCOrapid $^{\circledR}$ (Lidco Group Plc, London, UK) pulse contour analysis from the child's indwelling right axillary arterial catheter [1]. One minute of representative compression data was recorded from two rescuers, who compressed manually through a force-sensing mat on the child's sternum (Tekscan Inc ${ }^{\circledR}$, Boston MA, USA). The rescuers received no performance feedback above standard monitoring. Compressions were manually synchronised with the beat-to-beat haemodynamic data. Uni-variable and multivariable linear regression analyses of maximum force in newtons $\left(F_{\max }, \mathrm{N}\right)$, leaning force $\left(F_{\min }, \mathrm{N}\right)$ and time period between consecutive compressions (TP, s) were used to evaluate the effect of compressions on SVI and DBP.

A01

Rescuer 1 applied $51 \mathrm{~N}$ (SD $6 \mathrm{~N}$ ) mean force at a rate of 97 compressions per minute (cpm), Fig. 1a. Rescuer 2 delivered greater forces: $137 \mathrm{~N}$ (SD $15 \mathrm{~N})$ at $126 \mathrm{cpm}$. The second rescuer also maintained a larger $F_{\text {min }}$ mean $(\mathrm{SD}) 22 \mathrm{~N}(4 \mathrm{~N})$ versus $7 \mathrm{~N}(0.7 \mathrm{~N})$. Absolute forces were lower than reported with different technologies [23]. Maximum force and $F_{\min }$ demonstrated co-linearity, so change in force was calculated $\left(\delta F=F_{\max }-F_{\min }\right)$.

Fig. 1

a Chest compression force-time profile collected during an actual paediatric cardiac arrest. cpm compressions per minute. The thin Tekscan ${ }^{\circledR}$ mat was placed on the lower sternum to record manual force (in newtons) with each compression. A representation of the variables measured during compressions is included. The graph shows force measured over 
1 min of resuscitation by two rescuers. Rescuer 1 used lower forces and rate of compressions; rescuer 2 used both greater $F_{\max }$ and $F_{\min }$ at a faster rate. The child received noradrenaline and adrenaline during repeated arrests. The force data are limited to $1 \mathrm{~min}$ as the machine erroneously defaulted to a factory setting which allowed only this duration of data collection. b Relationship of stroke volume index with chest compression force during resuscitation. The broken horizontal line represents baseline, pre-arrest stroke volume index. Most compressions produced a stroke volume index greater than baseline, higher values being demonstrated by rescuer 2 . The LiDCOrapid ${ }^{\circledR}$ was calibrated $8 \mathrm{~h}$ before the arrest using the cardiac index value obtained with a suprasternal ultrasound cardiac output monitor (USCOM $^{\circledR}$, USCOM Ltd, NSW, Aus). Baseline SVI and DBP were determined retrospectively by analysing and averaging $1 \mathrm{~min}$ of data 1 and $2 \mathrm{~h}$ before the arrest occurred Baseline pre-arrest SVI was $31 \mathrm{~mL} / \mathrm{m}^{2}$ (SD $1.2 \mathrm{~mL} / \mathrm{m}^{2}$ ), Fig. 1b. During compressions, SVI increased with applied force. Mean (SD) SVIs for rescuer 1 and 2 were $34.5 \mathrm{~mL} / \mathrm{m}^{2}\left(1.5 \mathrm{~mL} / \mathrm{m}^{2}\right)$ and $46.1 \mathrm{~mL} / \mathrm{m}^{2}\left(10.6 \mathrm{~mL} / \mathrm{m}^{2}\right)$, respectively. On multivariable analysis, each additional newton of $\delta F$ increased SVI by $0.18 \mathrm{~mL} / \mathrm{m}^{2}(95 \%$ CI $0.09-$ $\left.0.27 \mathrm{~mL} / \mathrm{m}^{2}, p<0.001\right)$. TP did not predict SVI $(p=0.5)$.

Baseline pre-arrest DBP was $43 \mathrm{mmHg}$ (SD $1.4 \mathrm{mmHg}$ ). Mean (SD) DBPs for rescuers 1 and 2 were $15 \mathrm{mmHg}(1.8 \mathrm{mmHg})$ and $12 \mathrm{mmHg}(2.9 \mathrm{mmHg})$, respectively. On multivariable analysis, DBP rose by $19 \mathrm{mmHg}$ per second increase in TP (95\% CI 9$28 \mathrm{mmHg}, p<0.001)$, whereas $\delta F$ was not associated with DBP $(p=0.3)$.

These novel, surprising observations suggest the following: (a) wide ranges of forces were associated with SVI equivalent to or greater than the pre-arrest baseline. (b) Within the recommended rate range, rate did not affect SVI. (c) As rate slowed (TP increasing), so DBP rose, contrary to animal evidence [4]. This may reflect the higher $F_{\min }$ (indicating incomplete compression release) demonstrated by rescuer 2 .

Given this potential trade-off between rate and force in achieving optimal DBP and SVI, ideal values for paediatric rate and force are required [5]. This example merits future study, suggesting that in addition to rate and DBP, performance and potentially outcome may improve with additional real-time force and SVI feedback.

\section{Acknowledgements}

Participating researchers were funded by Sparks (Sport Aiding Medical Research for Kids) medical research charity \& the British Heart Foundation (BHF). The work was supported by the National Institute for Health Research Biomedical Research Centre at Great Ormond Street Hospital for Children NHS Foundation Trust and University College London. Our thanks also to the family and hospital staff who supported the research. The funding bodies had no role in the study design and execution, writing or submission of the manuscript. Compliance with ethical standards

Ethical approval 
Ethics approval was granted by the National Research Ethics Service Committee (registration number 12/LO/1700, protocol v.1), for recording compressions during CPR in a child in a tertiary paediatric hospital. Permission was granted to collect the data automatically during an arrest and to obtain retrospective written, informed consent from the family and from the rescuers to analyse and present the data.

Conflicts of interest

The authors declare they have no conflict of interest.

\section{References}

1 .

Asamoto M, Orii R, Otsuji M, Bougaki M, Imai Y, Yamada Y (2017) Reliability of cardiac output measurements using LiDCOrapid and FloTrac/Vigileo across broad ranges of cardiac output values. J Clin Monit Comput 31:709-716

2.

Gregson RK, Cole TJ, Skellett S, Bagkeris E, Welsby D, Peters MJ (2017) Randomised crossover trial of rate feedback and force during chest compressions for paediatric cardiopulmonary resuscitation. Arch Dis Child 102:403-409

3.

Brimacombe JM, Wilson DR, Hodgson AJ, Ho KC, Anglin C (2009) Effect of calibration method on Tekscan sensor accuracy. J Biomech Eng 131:034503 4.

Maier GW, Tyson GS Jr, Olsen CO, Kernstein KH, Davis JW, Conn EH, Sabiston DC Jr, Rankin JS (1984) The physiology of external cardiac massage: high-impulse cardiopulmonary resuscitation. Circulation 70:86-101

5.

Sutton RM, French B, Meaney PA, Topjian AA, Parshuram CS, Edelson DP, Schexnayder S, Abella BS, Merchant RM, Bembea M, Berg RA, Nadkarni VM, American Heart Association's Get With The Guidelines-Resuscitation I (2016) Physiologic monitoring of CPR quality during adult cardiac arrest: a propensity-matched cohort study. Resuscitation 106:76-82

\section{Proof Review}

No Correction 